\title{
MICROBIOTA AISLADA DEL PEPINO DE MAR (Isostichopus badionotus) NATIVO DE LA BAHÍA DE TAGANGA, CARIBE COLOMBIANO
}

\author{
MICROBIOTA ISOLATED FROM THE SEA CUCUMBER (Isostichopus badionotus) \\ NATIVE TO TAGANGA BAY, COLOMBIAN CARIBBEAN
}

Jorge Luna-Fontalvo, Adriana Rodríguez-Forero y Jonathan Sarmiento-Rodríguez

\begin{abstract}
RESUMEN
Se identificaron las comunidades bacterianas y fúngicas asociadas a la piel y al tracto digestivo de Isostichopus badionotus, con el objetivo de evaluar las posibles relaciones antagónicas entre éstas. Para tal fin, se realizaron frotis superficiales a nivel de la epidermis y el tracto digestivo, los cuales se sembraron en medios de cultivo específicos para bacterias y hongos. La identificación de los microorganismos se realizó teniendo en cuenta la morfología colonial en medio de cultivos y las características microscópicas. Así mismo, a las colonias bacterianas purificadas se les aplicaron pruebas bioquímicas a través de los micrométodos BBL Crystal ${ }^{\circledR}$ para Gram negativos y Gram positivos. Los hongos fueron identificados por medio de métodos microscópicos empleando claves taxonómicas. Se analizó la actividad inhibidora de las cepas bacterianas mediante la técnica de difusión en agar con discos de sensibilidad. En total se aislaron 42 cepas bacterianas de las cuales se seleccionaron diez para su identificación taxonómica: Bacillus cereus, B. subtilis, B. megaterium, Corynebacterium aquaticum, Proteus vulgaris, P. mirabilis, Enterobacter agglomerans, Serratia marcescens, Pseudomonas maltophilia y Acinetobacter anitratus. El enfrentamiento entre estas especies bacterianas no reflejó actividad inhibitoria en el crecimiento. Se aislaron 27 cepas fúngicas de las cuales se identificaron ocho especies: Penicillium sp., Paecilomyces sp., Aspergillus sp., Aspergillus niger, A. flavus, Gliocladium sp., Cladosporium fulvum y $C$. herbarum. Entre las especies fúngicas se logró observar un efecto inhibitorio en las pruebas de enfrentamiento dual. Las especies identificadas pueden corresponder a la microbiota normal del pepino como también estar presente en el agua o sedimento; por esa razón se requieren estudios complementarios que confirmen los resultados obtenidos.
\end{abstract}

Palabras Clave: bacterias, hongos, pepino de mar, interacciones microbianas

\begin{abstract}
The bacterial and fungal communities associated to the skin and digestive tract of Isostichopus badionotus were identified in order to evaluate the antagonistic interactions among them. For this purpose, surface smears were made to the epidermis and digestive tract, and this smears were grown in bacteria - and fungi - specific culture media. The identification of microorganisms was performed considering colonial morphology on culture media and microscopic features. Also, biochemical tests through BBL Crystal ${ }^{\circledR}$ micromethods for Gram negative and Gram positive bacteria were applied to the purified bacterial colonies. The fungi were identified through microscopic observations and using taxonomic keys. The bacterial strains' inhibitory activity was analyzed by the agar diffusion disk method. Results showed a total of 42 isolated bacterial strains from which ten species were selected for taxonomic identification: Bacillus cereus, B. subtilis, Bacillus megaterium, Corynebacterium aquaticum, Proteus vulgaris, P. mirabilis, Enterobacter agglomerans, Serratia marcescens, Pseudomonas maltophilia, and Acinetobacter anitratus. Confrontation among these bacterial species did not show growth inhibitory activity. A total of 27 fungal strains were isolated, from which eight species were identified: Penicillium sp., Paecilomyces sp., Aspergillus sp., Aspergillus niger, A. flavus, Gliocladium sp., Cladosporium fulvum and C. herbarum. Fungal species showed inhibitory effects in dual confrontation tests. The identified species could correspond to the normal microbiota of the cucumber or to the microbiota associated to the water and/or the sediment, thus further studies are needed to confirm the obtained results.
\end{abstract}

Keywords: bacteria, fungi, sea cucumber, microbial interactions 


\section{INTRODUCCIÓN}

El pepino de mar, o su forma seca (beche de mer) es comercializado desde hace siglos en Asia y algunos países de Europa como un producto para la alimentación por su alto contenido proteico, como afrodisíaco y para tratamientos farmacéuticos y medicinales, siendo considerado como el gingseng del mar. Se ha reportado que tiene propiedades para cicatrización de heridas y el tratamiento de úlceras de estómago, asma, hipertensión, reumatismo y sinusitis, entre otros (Aminin et al., 2010; Mayer et al., 2011). Se han descrito algunas propiedades como las de poseer mucotin, una proteína que podría retardar el envejecimiento, actuar como antitumoral y como agente en la coagulación de la sangre además la presencia de lectina inhibe el desarrollo de posibles parásitos, en adición a algunas propiedades antibacterianas y antifúngicas (Ridzwan et al., 1995; Haug et al., 2002; Farouk et al., 2007; Kumar et al., 2007; Ismail et al., 2008). Es posible obtener ciertos tipos de metabolitos o compuestos bioactivos importantes en la industria alimentaria, química y farmacéutica de gran interés comercial, tales como el triterpeno glucósido, el sulfato de condroitin, los glucosaminoglucanos, los polisacáridos sulfatados, los esteroles, los compuestos fenólicos, los péptidos y las lectinas (Kerr y Chen, 1995; Vieira et al., 1999; Mourao y Pereira, 1999; Bakunina et al., 2000; Miyamoto et al., 2006; Aminin et al., 2010).

Además de lo anterior, los pepinos de mar representan un importante papel ecológico en el ambiente marino, pues al alimentarse de sedimento se benefician de la materia orgánica, los microorganismos y nutrientes circulantes y, a su vez, oxigenan el sedimento al removerlo, proveen hasta el doble de nitrógeno, evitan su estratificación y procesan la materia orgánica desprendida de plantas fanerógamas (Sambrano et al., 1990; Tagliafico et al., 2011). Dentro de las interacciones ecológicas que existen entre los organismos marinos (crustáceos, moluscos, equinodermos, poliquetos, etc.) con los microorganismos (bacterias y hongos), se encuentran las interacciones positivas (simbiosis, protocooperación, comensalismo) y negativas (competencia, parasitismo, amensalismo) (Schlegel, 1997; Madigan et al., 2003). Así mismo, dentro de las interacciones negativas surge el antagonismo o control biológico entre microorganismos de un determinado huésped. Estudios realizados por Mora-Cristancho et al. (2009) sobre la actividad antagónica entre bacterias aisladas de esponjas de mar, mencionan que las relaciones antagónicas influyen como un posible mecanismo de regulación del crecimiento poblacional de microorganismos o ataques frente a patógenos.

En Colombia existen numerosas especies de pepino de mar habitantes de aguas someras y profundas, que están siendo explotadas ilegalmente. La ausencia de información sobre holoturios es un tópico constante en el ámbito científico y académico nacional. Especies marinas muy importantes por su papel en la ecología del sistema son a menudo subvaloradas y de ellas se desconoce su significado biológico y potencial en los campos de aplicación práctica como la farmacología, la química, la acuacultura o la alimentación. Un interés creciente sobre el estudio de estos invertebrados marinos, que actúan como "bioturbinas", motivó al estudio de la composición de su microbiota asociada a la piel y al tubo digestivo, teniendo en cuenta la hipótesis de que los grupos bacterianos y fúngicos aislados del pepino de mar, a nivel de la piel (superficie dorsal y ventral) y el tracto digestivo, son colonizados por sus similares que ejercen un control en el crecimiento de las poblaciones de otras bacterias y hongos. Se planteó entonces el objetivo de identificar las comunidades bacterianas y fúngicas asociadas al pepino de mar Isostichopus badionotus, especie de importancia comercial y nativa de las aguas someras del Caribe colombiano, así como evaluar las interacciones antagónicas entre dichas comunidades.

\section{MATERIALES Y MÉTODOS}

\section{Obtención de las muestras}

Se colectaron ocho especímenes de pepino de mar Isostichopus badionotus provenientes de la zona ubicada frente al Aeropuerto Simón Bolívar $\left(11^{\circ} 07^{\prime} 10.74^{\prime \prime} \mathrm{N}\right.$ y $\left.74^{\circ} 13^{\prime} 50.33^{\prime \prime} \mathrm{O}\right)$ y la Bahía de Taganga $\left(11^{\circ} 16.04^{\prime \prime} \mathrm{N}\right.$, $74^{\circ} 11.24^{\prime \prime} \mathrm{W}$ ), las cuales fueron transportados vivas al Laboratorio de Microbiología de la Universidad del Magdalena, para su procesamiento inmediato.

\section{Procesamiento de muestras y aislamiento de bacterias y hongos}

En el laboratorio, los pepinos fueron sacrificados mediante hipotermia y lavados tres veces con $10 \mathrm{ml}$ de agua de mar esterilizada (AME). El aislamiento de microorganismos se realizó a partir de la piel y el tubo digestivo de cada individuo, utilizando la técnica de barrido con hisopado por duplicado para remover las bacterias y hongos adheridos a la superficie. El 
hisopo se sembró por duplicado en placas Petri (10 $\mathrm{cm}$ ) con medio Agar Glicerol Agua de Mar (AGAM) y en Agar Malta Sal (AMS) al 5 \% para el aislamiento de bacterias y hongos, respectivamente. Los cultivos se incubaron a $25 \pm 2{ }^{\circ} \mathrm{C}$ en oscuridad y bajo condiciones aeróbicas y microaerofílicas durante 7 días con registro de observaciones diarias. Se recolectaron las colonias heterotróficas tomando como criterio el buen crecimiento en el medio de aislamiento.

Las cepas bacterianas obtenidas fueron caracterizadas teniendo en cuenta los diferentes morfotipos de las colonias aisladas (Gómez et al, 2006) y con base en la tinción de Gram, empleando un microscopio óptico. Para su identificación se realizaron pruebas bioquímicas mediante los micrométodos de BBL Crystal ${ }^{\circledR}$ para Gram negativos y Gram positivos. Para los hongos se realizaron caracterizaciones microscópicas y macroscópicas de las colonias aisladas. Adicionalmente, se efectuaron ensayos con microcultivos para inducir a la formación de estructuras de reproducción sexual y asexual. La identificación de los géneros y especies se realizó mediante las claves de VonArx (1981) y Barnett y Hunter (1987).

\section{Ensayos de antagonismo bacteriano in vitro}

A partir de las cepas bacterianas aisladas e identificadas, se seleccionaron las más frecuentes para realizar los ensayos de antagonismo mediante la técnica de difusión en agar con discos de sensibilidad (Hewitt y Vicentt, 1989). Para tal fin, se realizaron cultivos con las cepas bacterianas seleccionadas en caldo con la composición del medio sólido AGAM (Smith y Hayasaka, 1982), a $25 \pm 2{ }^{\circ} \mathrm{C}$ durante 24 horas; se inoculó en la placa Petri con medio AGAM $100 \mu \mathrm{l}$ de cada cepa bacteriana y se ajustó al patrón de turbidez No. 0.5 de McFarland, equivalente a $1 \times 10^{8}$ células $/ \mathrm{ml}$ (Koneman et al., 1987). En este caso se inocularon en los discos de papel $20 \mu \mathrm{l}$ de cada una de las cepas bacterianas (Hewitt y Vincent, 1989), cuya capacidad antagónica se ensayó sin ajuste de la concentración por el patrón de MacFarland (Brinkhoff et al., 2004). Para la determinación de los enfrentamientos entre las cepas bacterianas se utilizó un disco de antibiótico de amplio espectro (Sulfametoxasol - SXT) con una concentración de $25 \mu \mathrm{g}$ (control positivo) y un disco estéril de papel sin antibiótico y sin caldo marino (control negativo). La presencia de halos de inhibición alrededor de los discos fue considerada como una respuesta cualitativa antagónica entre las cepas. Cada prueba fue realizada por triplicado.

\section{Pruebas de enfrentamiento dual de las cepas fúngicas}

Los ensayos antagónicos entre las cepas de hongos fueron realizados mediante la técnica de enfrentamiento dual en placas con medio de cultivo AMS (5 \%) (Pérez et al., 2002). Para esto, y de forma separada cada una de las cepas fueron enfrentadas entre ellas mismas, inoculándolas a $4 \mathrm{~cm}$ de distancia para ser reportado como crecimiento influenciado. Al mismo tiempo, cada uno de los inóculos de las cepas identificadas fue colocado en el centro de otra placa con el mismo medio de cultivo (crecimiento libre). Como control positivo se utilizó una cepa de Trichoderma sp. (hongo antagónico, tomado del Laboratorio de Microbiología de la Universidad del Magdalena). Los cultivos fueron incubados a $25{ }^{\circ} \mathrm{C}$ por 7 días. Finalizado el tiempo de incubación, se calculó el porcentaje de Inhibición Micelial (\% IM) de acuerdo con la siguiente fórmula:

$$
\% \mathrm{IM}=[(\mathrm{Cl}-\mathrm{Ci}) * 100]
$$

Donde $\mathrm{Cl}$ es el crecimiento micelial libre y $\mathrm{Ci}$ es el crecimiento micelial influenciado (Suárez-Contreras y Rangel-Riaño, 2008). El porcentaje de inhibición del crecimiento micelial (\% IM) fue determinado para cada una de las cepas usando el modelo matemático recomendado por Pérez et al. (2002).

\section{RESULTADOS}

\section{Aislamiento y evaluación de actividad antimicrobiana entre especies bacterianas}

Se aislaron un total de 42 cepas bacterianas asociadas a I. badionotus, de las cuales se seleccionaron 10 para su identificación taxonómica y ensayos antagónicos. En la Tabla 1 se muestran las especies identificadas, así como la zona del cuerpo del pepino de mar de la cual fueron aisladas.

Con excepción de Proteus mirabilis, la mayoría de las cepas bacterianas mostraron sensibilidad ante el antibiótico SXT (Sulfametoxasol). Los ensayos antagónicos determinaron que las especies bacterianas aisladas no presentan actividad inhibitoria, pues no se observaron halos de inhibición en el crecimiento al enfrentar cada una de estas con las otras especies bacterianas. 
Tabla 1. Especies bacterianas aisladas del pepino de mar Isostichopus badionotus en la Bahía de Taganga, Caribe colombiano.

\begin{tabular}{ll}
\hline \multicolumn{1}{c}{ Cepa } & Zona de aislamiento \\
\hline $\begin{array}{l}\text { Bacillus cereus } \\
\text { Bacillus subtilis } \\
\text { Bacillus megaterium }\end{array}$ & Piel (dorso) \\
\hline $\begin{array}{l}\text { Corynebacterium acuaticum } \\
\text { Proteus vulgaris }\end{array}$ & Piel (dorso) \\
\hline Enterobacter agglomerans & Tracto digestivo \\
\hline $\begin{array}{l}\text { Proteus mirabilis } \\
\text { Serratia marcescens }\end{array}$ & Piel (ventral) \\
Stenotrophomonas maltophilia & \\
\hline Acinetobacter anitratus & Tracto digestivo \\
\hline
\end{tabular}

\section{Aislamiento y actividad antagónica de hongos asociados al pepino de mar}

Se aislaron 27 cepas fúngicas de las distintas partes de los pepinos. Todas las cepas aisladas corresponden a hongos de talo micelial pertenecientes al grupo de los hongos Mitospóricos (Deuteromycotas o grupo anamórfico de Ascomycetes y Basidiomycetes) (Hawksworth et al., 1995; Alexopoulos et al., 1996). Del total de las cepas se lograron identificar ocho especies fúngicas (Tabla 2).

Tabla 2. Especies de hongos aislados del pepino de mar Isostichopus badionotus en la Bahía de Taganga, Caribe colombiano.

\begin{tabular}{ll}
\hline \multicolumn{1}{c}{ Cepa } & \multicolumn{1}{c}{ Zona de aislamiento } \\
\hline Aspergillus flavus & Piel (dorso) \\
Aspergillus niger & Piel (ventral) - Tracto digestivo \\
Aspergillus sp. & Piel (ventral) \\
Cladosporium fulvum & Piel (dorso y ventral) \\
Cladosporium herbarum & Tracto digestivo \\
Gliocladium sp. & Tracto digestivo \\
Paecilomyces & Piel (ventral) - Tracto digestivo \\
& Piel (dorsal - ventral) - tracto \\
Penicillium sp. & digestivo \\
\hline
\end{tabular}

\section{Ensayos antagónicos mediante enfrentamiento dual}

Cada una de las cepas fúngicas señaladas en la Tabla 2 fueron enfrentadas entre sí para establecer si existía actividad antagónica. Así mismo, en la Tabla 3 se muestran los códigos asignados a cada una de las cepas fúngicas con los diámetros de la colonia considerados como crecimiento libre. Los códigos se asignaron con el fin de identificar las cepas al momento de realizar los cálculos matemáticos de enfrentamiento dual.

Tabla 3. Crecimiento libre de las cepas fúngicas de pepino de mar Isostichopus badionotus en la Bahía de Taganga, Caribe colombiano.

\begin{tabular}{clr}
\hline Código & \multicolumn{1}{c}{ Cepa } & Crecimiento libre (cm) \\
\hline A & Penicillium sp. & 3.4 \\
B & Paecilomyces sp. & 7.0 \\
C & Aspergillus sp. & 9.0 \\
D & Aspergillus niger & 5.0 \\
E & Aspergillus flavus & 4.0 \\
F & Gliocladium sp. & 7.6 \\
G & Cladosporium fulvum & 8.5 \\
H & Cladosporium herbarum & 2.1 \\
I & Trichoderma sp. & 10.0 \\
\hline
\end{tabular}

El enfrentamiento entre las especies de hongos aisladas del pepino de mar produjo comportamientos muy variables en la inhibición del crecimiento (Tabla 4), pudiéndose observar desde un efecto nulo (0 \%) (C vs $H$; $D$ vs $A$; $D$ vs $C$; $D$ vs $E$; $D$ vs $H$; $H$ vs $G$ ) hasta un alto porcentaje de inhibición micelial entre los enfrentamientos de C vs F $(94,4 \%)$, C vs G $(91,1 \%)$ y G vs F $(84,7$ \%).

La cepa I (Trichoderma, hongo antagónico) fue considerada como control positivo, presentando el mayor diámetro de colonia en el crecimiento libre. El porcentaje de inhibición mínimo frente al hongo antagónico fue de $45 \%$ (I vs A) mientras que el mayor porcentaje de inhibición micelial fue de I vs C. 
Tabla 4. Actividad antagónica entre las especies fúngicas aisladas de pepino de mar Isostichopus badionotus.

\begin{tabular}{|c|c|c|c|c|}
\hline $\begin{array}{c}\text { Cepa } \\
\text { enfrentada }\end{array}$ & Cepa & MB (cm) & MA (cm) & $\% \mathrm{Ml}$ \\
\hline \multirow{7}{*}{ A } & B & 3,4 & 1,3 & 61,8 \\
\hline & C & 3,4 & 1,5 & 55,9 \\
\hline & $\mathrm{D}$ & 3,4 & 1,0 & 70,6 \\
\hline & E & 3,4 & 1,5 & 55,9 \\
\hline & $\mathrm{F}$ & 3,4 & 2,5 & 26,5 \\
\hline & G & 3,4 & 1,7 & 50,0 \\
\hline & $\mathrm{H}$ & 3,4 & 2,3 & 32,4 \\
\hline \multirow{7}{*}{ B } & A & 7,0 & 4,0 & 42,9 \\
\hline & C & 7,0 & 5,0 & 28,6 \\
\hline & D & 7,0 & 4,5 & 35,7 \\
\hline & E & 7,0 & 4,5 & 35,7 \\
\hline & $\mathrm{F}$ & 7,0 & 4,5 & 35,7 \\
\hline & G & 7,0 & 3,4 & 51,4 \\
\hline & $\mathrm{H}$ & 7,0 & 3,0 & 57,1 \\
\hline \multirow{7}{*}{ C } & A & 9,0 & 4,0 & 55,6 \\
\hline & B & 9,0 & 2,0 & 77,8 \\
\hline & D & 9,0 & 2,0 & 77,8 \\
\hline & E & 9,0 & 2,0 & 77,8 \\
\hline & $\mathrm{F}$ & 9,0 & 0,5 & 94,4 \\
\hline & G & 9,0 & 0,8 & 91,1 \\
\hline & $\mathrm{H}$ & 9,0 & 9,0 & 0,0 \\
\hline \multirow{7}{*}{$\mathrm{D}$} & A & 5,0 & 5,0 & 0,0 \\
\hline & B & 5,0 & 3,5 & 30,0 \\
\hline & C & 5,0 & 5,0 & 0,0 \\
\hline & $\mathrm{E}$ & 5,0 & 5,0 & 0,0 \\
\hline & $\mathrm{F}$ & 5,0 & 4,5 & 10,0 \\
\hline & G & 5,0 & 4,5 & 10,0 \\
\hline & $\mathrm{H}$ & 5,0 & 5,0 & 0,0 \\
\hline \multirow{7}{*}{ E } & A & 4,0 & 3,5 & 12,5 \\
\hline & B & 4,0 & 1,5 & 62,5 \\
\hline & C & 4,0 & 3,6 & 10 \\
\hline & D & 4,0 & 2,7 & 32,5 \\
\hline & $\mathrm{F}$ & 4,0 & 1,7 & 57,5 \\
\hline & G & 4,0 & 3,2 & 20 \\
\hline & $\mathrm{H}$ & 4,0 & 2,4 & 40 \\
\hline
\end{tabular}

\begin{tabular}{|c|c|c|c|c|}
\hline $\begin{array}{c}\text { Cepa } \\
\text { enfrentada }\end{array}$ & Cepa & $\mathrm{MB}(\mathrm{cm})$ & MA (cm) & $\% \mathrm{MI}$ \\
\hline \multirow{7}{*}{ F } & A & 7,6 & 4,3 & 43,4 \\
\hline & B & 7,6 & 1,6 & 78,9 \\
\hline & c & 7,6 & 1,0 & 86,8 \\
\hline & D & 7,6 & 2,8 & 63,2 \\
\hline & E & 7,6 & 7,0 & 7,9 \\
\hline & G & 7,6 & 5,5 & 27,6 \\
\hline & $\mathrm{H}$ & 7,6 & 3,7 & 51,3 \\
\hline \multirow{7}{*}{ G } & A & 8,5 & 5,1 & 40,0 \\
\hline & B & 8,5 & 2,0 & 76,5 \\
\hline & c & 8,5 & 8,4 & 1,2 \\
\hline & D & 8,5 & 1,8 & 78,8 \\
\hline & E & 8,5 & 2,6 & 69,4 \\
\hline & F & 8,5 & 1,3 & 84,7 \\
\hline & $\mathrm{H}$ & 8,5 & 5,1 & 40,0 \\
\hline \multirow{7}{*}{$\mathrm{H}$} & A & 2,1 & 1,5 & 28,6 \\
\hline & B & 2,1 & 0,8 & 61,9 \\
\hline & c & 2,1 & 0,5 & 76,2 \\
\hline & D & 2,1 & 1,5 & 28,6 \\
\hline & E & 2,1 & 1,5 & 28,6 \\
\hline & F & 2,1 & 1,5 & 28,6 \\
\hline & G & 2,1 & 2,1 & 0,0 \\
\hline
\end{tabular}

\%IM: Porcentaje de inhibición Micelial, $\mathrm{C}_{\mathrm{I}}$ : crecimiento micelial influenciado, $\mathrm{M}_{\mathrm{L}}$ : crecimiento micelial libre (control)

\section{DISCUSIÓN}

Los holoturios se encuentran dentro los organismos más pobremente estudiados en el mundo. Se conoce que son consumidores de sedimentos y elementos en suspensión, y gracias a ellos es posible "oxigenar" el substrato, mover la arena y reciclar el detritus (Sant, 2006). Su papel como "bioturbinas" probablemente esté relacionado con la microbiota presente en la piel y órganos internos y con la capacidad de utilizar la materia orgánica a través de procesos internos de quimiosíntesis (Ward-Rainey et al., 1996). 
Se aislaron 69 cepas microbianas, de las cuales 10 corresponden a especies bacterianas y 8 a especies fúngicas, que se consideran como microbiota presente en piel y tubo digestivo de I. badionotus, especie habitante de las aguas costeras del Caribe colombiano. Estos hallazgos proporcionan información valiosa relacionada con la microbiología, la biología y la ecología de la especie, ya que puede explicar de cierta manera el comportamiento de "bioturbina" por el cual son conocidos estos invertebrados, al transformar material orgánico en forma de grano y soltar al agua en forma de nutrientes. Además, al cavar el fondo continuamente esparcen el sustrato a su alrededor (Nithyanandan, 2003). Las bacterias y hongos asociados al cuerpo de los pepinos son cosmopolitas; también constituyen la microbiota del sedimento que remueven los pepinos para obtener sus nutrientes, y muchos de ellas contribuyen a descomponer la materia orgánica (Roberts et al., 2001).

Por otro lado, los microorganismos desempeñan un papel importante en la vida de sus huéspedes y en los ecosistemas en que habitan, al actuar como parásitos o simbiontes comensales. En este caso, los microorganismos marinos identificados son esenciales en los ecosistemas que habitan los pepinos de mar, pues influyen en el reciclaje de nutrientes, la remineralización de las fuentes de carbono absorbidas, el movimiento de materia y energía en su medio, la descomposición del detritus, la dieta de organismos bentónicos o en las actividades metabólicas normales de los individuos, además de actuar como una barrera defensiva a nivel de la dermis (Liberra y Lindeguist, 1995; Ward-Rainey et al., 1996).

Sobre la diversidad y estructura de la comunidad de la microbiota de la piel y el tubo digestivo de los holoturios, es poco lo que se ha estudiado y aún se desconocen muchos aspectos relacionados con los procesos y funciones que éstas ejercen en la digestión de nutrientes. Así, algunos autores han descrito diversos métodos para el análisis y cultivo de la microbiota, y exponen conceptos y documentan especies bacterianas, hongos y agentes antibacteriales (Ridzwan et al., 1995; Ward-Rainey et al., 1996; Kumar et al., 2007; Ramírez-Gómez et al., 2010; Mayer et al., 2011). En ese sentido, Ward-Rainey et al. (1996) realizaron estudios 80 de la microbiota en Holothuria atra mediante análisis de secuencia del $16 \mathrm{~S}$ ADNr con muestras de tubo digestivo y sedimento. Se encontraron 24 cepas relacionadas con el género Vibrio, otras con Bacillus, las subclases alfa y gama de Proteobacterias, el linaje de Cytophaga - Flavobacterium
- Bacteroides y otros Actinomycetales. Por otro lado, se ha identificado la microbiota aislada del tubo digestivo de Asterechinus elegans (Echinodermata), empleando técnicas de biología molecular, encontrando Proteobacteria, Planctomycete, Firmicutes, Cytophaga-FlexibacterBacteroides y Actinobacteria (Becker et al., 2009).

A pesar de que la metodología empleada para el aislamiento e identificación de las cepas bacterianas se basó en características bioquímicas y microscópicas, se logró identificar especies de las familias Enterobacteriaceae, Bacillaceae y Corynebacteriaceae aislados de I. badionotus, lo que demuestra su diversidad y su posible desempeño en la defensa del pepino de mar ante agresores externos que intenten penetrar su tegumento, así como en los procesos metabólicos de absorción, asimilación, digestión enzimática y descomposición de nutrientes en el intestino. Estas afirmaciones pueden estar en concordancia con lo planteado previamente (Purser, 1970; Deming et al., 1981; Deming y Colwell, 1982).

Una de las funciones ecológicas que cumplen los microorganismos en los ecosistemas es la de interactuar con el medio y con todos los organismos. Las interacciones microbianas pueden ser de forma neutra (neutralismo), positiva (comensalismo, simbiosis, mutualismo, protocooperación) o negativa (competencia, amensalismo, parasitismo y depredación) (Schlegel, 1997; Madigan et al., 2003). Los ensayos realizados con los microorganismos aislados tanto de la epidermis como del tracto digestivo del pepino de mar fueron sometidos a pruebas de interacción negativa mediante enfrentamientos (antagonismo), es decir, se quiso evaluar si el contacto directo entre las cepas bacterianas seleccionadas causaría un efecto inhibitorio en el crecimiento de al menos una de las cepas; en los resultados no se evidenció efecto inhibitorio. Por esta razón, la mayoría de las especies bacterianas encontradas podrían estar interactuando de alguna manera neutra u oportunista a través de una función beneficiosa, o sistema de defensa para los organismos invasores. Sin embargo, no se podría afirmar si las especies aisladas del pepino de mar correspondan a microorganismos benéficos o perjudiciales, a pesar que algunos trabajos sobre microorganismos aislados de holotúridos mencionan que éstos han sido utilizados como productos farmacéuticos (probióticos), terapéuticos o antimicrobianos (Ridzwan et al., 1995; Ward-Rainey et al., 1996; Kumar et al., 2007; Wang et al., 2008; Ramírez-Gómez et al., 2010; Mayer et al., 2011). 
Por otro lado, la técnica de enfrentamiento dual aplicada para evaluar la interacción entre las cepas fúngicas es una técnica que demuestra la capacidad que tienen algunas especies de hongos para reprimir el crecimiento de otros mohos al momento de ser enfrentados (Antagonismo o control biológico). Esta acción es involuntaria y se da por la producción de sustancias o compuestos derivados del metabolismo (enzimas, toxinas, antibióticos, ácidos, bacteriocinas, etc.) que causan daño al microorganismo enfrentado.

La cepa de Trichoderma sp., utilizada como control positivo enfrentada a las cepas de hongos aisladas del pepino de mar, es un hongo antagónico, cuyo modo de acción se basa en la producción de enzimas extracelulares tales como quitinasas y celulasas, y se corresponden con la composición y estructura de las paredes celulares de los hongos (Infante et al., 2009). Los ensayos del enfrentamiento con las especies de los géneros Penicillium, Aspergillus, Gliocladium y Cladosporium demostraron una actividad inhibitoria en el crecimiento de cada una de estas cepas. Estos hongos corresponden a microorganismos oportunistas de cualquier sustrato u hospedero, saprófitos y cosmopolitas, relacionados con la descomposición y mineralización de la materia orgánica tanto de origen terrestre como acuático (Valenzuela, 2006).

\section{CONCLUSIONES}

A partir de la piel y tubo digestivo del pepino de mar (I. badionotus) se aisló una amplia variedad de microorganismos, incluyendo 42 cepas bacterianas. Diez de estas se hallaron indistintamente en piel y tubo digestivo: Bacillus cereus, B. subtilis, B. megaterium, Corynebacterium acuaticum, Proteus vulgaris, P. mirabilis, Enterobacter agglomerans, Serratia marcescens, Stenotrophomonas maltophilia y Acinetobacter anitratus. Los ensayos de antagonismo determinaron que las especies bacterianas aisladas no presentan actividad antagónica, al no observarse halos de inhibición.

De las 27 cepas fúngicas aisladas se lograron identificar ocho especies: Aspergillus niger, A. flavus, Aspergillus. sp., Cladopsorium fulvum, C. herbarum, Gliocladium sp., Paecilomyces sp., Penicillium sp. y Trichoderma sp. Los ensayos de enfrentamiento dual indicaron que las cepas de Paecilomyces sp. y Aspergillus niger tienen mejor actividad antagónica que las otras cepas fúngicas encontradas.
Deben efectuarse estudios moleculares y filogenéticos para confirmar la identificación de las especies bacterianas y fúngicas aisladas y adicionalmente continuar con la realización de ensayos antagónicos que permitan verificar su interacción.

\section{AGRADECIMIENTOS}

Este trabajo fue financiado por el Fondo Patrimonial de la Universidad del Magdalena, FONCIENCIAS de la Vicerrectoría de Investigación. Al personal que labora en el Laboratorio de Microbiología de la Universidad del Magdalena por el apoyo en esta investigación.

\section{BIBLIOGRAFÍA}

Alexopoulos, C., C. Mims y M. Blackwell. 1996. Introductory Mycology. 4 Ed., Jonn Wiley \& Sons. New York, USA. 235 p.

Aminin, D., E. Cha ykina, I. Agafonova, S. Avilov, V. Kalinin y V. Stonik. 2010. Antitumor activity of the immunomodulatory lead Cumaside. International Immunopharmacology 10(6): 648-654.

Barnett, H. y B. Hunter. 1987. Ilustrated Genera of Imperfect Fungi. 4 Ed. Macmillan Publishing Company. Minneapolis, Minnesota, USA. 218 p.

Bakunina, I., L. Shevchenko, O. Nedashkovskaya, N. Shevchenko, S. Alekseeva, V. Mikhailov y T. Zvyaginseva. 2000. Screening of marine bacteria for fucoidanases. Microbiology 69(3): 303-308.

Becker, P., S. Samadi, M. Zbinden, P. Compère y C. De Ridder. 2009. First insights into the gut microbiota associated with an echinoid from wood falls environments. Cahiers of Marine Biology 50(1): 343-352.

Brinkhoff, T., G. Bach, T. Heidon, L. Liang, A. Schlingloff y M. Simon. 2004. Antibiotic production by a Roseobacter clade-affiliated species from the German wadden sea and its antagonistic effects on indigenous isolates. Applied and Environmental Microbiology 70(4): 2560-2565.

Deming, J y R. Colwell. 1982. Barophilic bacteria associated with digestive tracts of abyssal holothurians. Applied and Environmental Microbiology 44(5): 1222-1230.

Deming, J., P. Tabor y R. Colwell. 1981. Barophilic growth of bacteria from intestinal tracts of deep-sea invertebrates. Microbial Ecology 7(1): 85-94. 
Farouk, A., F. Ghouse y B. Ridzwan. 2007. New bacterial species isolated from Malaysian sea cucumbers with optimized secreted antibacterial activity. American Journal of Biochemistry and Biotechnology 3(2): 60-65.

Gómez, M., L. Vivas, R. Ruíz, V. Reyes y C. Hurtado. 2006. Bacterias marinas nativas degradadoras de compuestos orgánicos persistentes en Colombia. Instituto de Investigaciones Marinas y Costeras - INVEMAR. Colombia. $32 \mathrm{p}$.

Haug, T., A. Kjuul, O. Styrvold, E. Sandsdalen, Ø. Olsen y K. Stensvåg. 2002. Antibacterial activity in Strongylocentrotus droebachiensis (Echinoidea), Cucumaria frondosa (Holothuroidea), and Asterias rubens (Asteroidea). Journal of Invertebrate Pathology 81(2): 94-102.

Hawksworth, D., P Kirk, B. Sutton y D. Pegler. 1995. Ainsworth \& Bisby's Dictionary of the Fungi. 8 Ed. CAB International. Cambridge, Inglaterra, $616 \mathrm{p}$.

Hewitt, W. y S. Vincent. 1989. The agar diffusion assay. pp 38-79. En: Hewitt, W. y S. Vicent (Eds). Theory and Application of Microbiological Assay, Academic Press, San Diego. 323 p.

Infante, D., B. Martínez, N. González y Y. Reyes. 2009. Mecanismos de acción de Trichoderma frente a hongos fitopatógenos. Revista de Protección Vegetal 24(1): 14-21.

Ismail, H., S. Lemriss, Z. Ben-Aoun, L. Mhadhebi, A. Dellai, Y. Kacem, P. Boiron y A. Bouraoui. 2008. Antifungal activity of aqueous and methanolic extracts from the Mediterranean sea cucumber, Holothuria polii. Journal of Medical Mycology 18(1): 23-26.

Kerr, R. y Z. Chen. 1995. In vivo and in vitro biosynthesis of saponins in sea cucumbers (Holothuroidea). Jorunal of Natural Products. 58(2): 172-176.

Koneman, E., S. Allen y V. Dowell, 1987. Sommers H. 1987. Diagnóstico Microbiológico. Texto y Atlas en Color. Médica Panamericana, Buenos Aires, Argentina. 1691 p.

Kumar, R., A. Chaturvedi, P. Shukla y V. Lakshmi. 2007. Antifungal activity in triterpene glycosides from the sea cucumber Actinopyga lecanora. Bioorganic and Medical Chemestry Letters 17(15): 4387-4391.

Liberra, K. y C. Lindeguist. 1995. Marine fungi: a prolific resource of biologically active natural products? Pharmazie 50(9): 583-588.

Madigan, M., J. Martinko, J. Perker y M. Sanchez. 2003. Brock Biología de los microorganismos. 10 Ed. Pearson Prentice Hall. España. 1096 p.
Mayer, A., A. Rodríguez, A. Berlinck y N. Fusetani. 2011. Marine pharmacology in 2007-8 Marine compounds with antibacterial, anticoagulant, antifungal, anti-inflammatory, antimalarial, antiprotozoal, antituberculosis, and antiviral activities; affecting the immune and nervous system, and other miscellaneous mechanisms of action. Comparative Biochemistry Physiology Part C 153: 191-222.

Miyamoto, T., K. Togawa, R. Higuchi, T. Komori y T. Sasaki. 2006. Constituents of Holothuroidea, II. Six newly identified biologically active triterpenoid glycoside sulfates from the sea cucumber Cucumaria echinata. European Journal of Organic Chemistry 1990(5): 453-460.

Mora-Cristancho, J., S. Zea-Sjoberg y D. Gil-Agudelo. 2009. Actividad antagónica entre bacterias epibióticas aisladas de esponjas marinas del caribe colombiano y su relación con la macroepibiosis. Boletín de Investigaciones Marinas y Costeras 38(1): 25- 38.

Mourao, P. y M. Pereira. 1999. Searching for alternatives to heparin: Sulfated fucans from marine invertebrates. Trends in Cardiovascular Medicine 9(8): 225-232.

Pérez, L., C. Ramírez, M. Martínez y N. Algecira. 2002. Efecto de las variables, condiciones de la fermentación y del sustrato en la producción de Trichoderma harzianum. Revista de Protección Vegetal 17(2): 1 - 142.

Purser, D. 1970. Nitrogen metabolism in the rumen: microorganisms as a source of protein for the ruminant animal. Journal of Animal Science 30(6): 988-1001.

Ramírez-Gómez, F., F. Aponte-Rivera, L. Méndez-Castaner y J. García-Arrarás. 2010. Changes in holothurians coelomocyte populations following immunestimulation with different molecular patterns. Fish and Shellfish Immunology 29(2): 175-185.

Ridzwan, B., M. Kaswandi, Y. Azman y M. Fuad. 1995. Screening for antibacterial agents in three species of sea cucumbers from coastal areas of sabah. General Pharmacology 7(26): 1539-1543.

Roberts, D., H. Moore, J. Berges, J. Patching, M. Carton y Eardly, D., 2001. Sediment distribution, hydrolytic enzyme profiles and bacterial activities in the guts of Oneirophanta mutabilis, Psychropotes longicauda and Pseudostichopus villosus: what do they tell us about digestive strategies of abyssal holothurians? Progress in Oceanography 50(1): $443-458$.

Sambrano, A., H. Díaz y J. Conde. 1990. Caracterización de la ingesta en Isostichopus badionotus (Selenka) y Holothuria mexicana Ludwig (Echinodermata: Holothuroidea). Caribbean Journal of Science 26(1): 45-51. 
Sant, G. 2006. Cites and Sea Cucumbers. pp 104 - 121. En: AW. Bruckner (Ed). Proceedings of the CITES Workshop on the Conservation of Sea Cucumbers in the Families Holothuriidae and Stichopodidae. Kuala Lumpur, Malaysia. NOAA Technical Memorandum, NMFS-OPR. 244p.

Schlegel, H. 1997. Microbiología General, 7 Ed., Omega S.A. Barcelona, España. 669 p.

Smith, G. y S. Hayasaka. 1982. Nitrogeiiase activity associated with Holodule wrightii roots. Applied Environmental Microbiology 43(6): 1244-1248.

Suárez-Contreras, L. y A. Rangel-Riaño. 2013. Aislamiento de microorganismos para control biológico de Moniliophthora roreri. Acta Agronómica 62(4): 370-378.

Tagliafico, A., M. Rangel y N. Rago. 2011. Distribución y densidad de dos especies de holoturoideos en la isla de Cubagua, Venezuela. Revista de Biología Tropical 59(2): 843-852
Valenzuela, E. 2006. Guía de Hongos. Universidad Austral de Chile. Valdivia, Chile. 51p.

Vieira, R., B. Mulloy y P. Mourão. 1999. Structure of a fucose-branched chondroitin sulphate from sea cucumber. Evidence for the presence of 3-0-sulfo- $\beta$-D-glucuronosyl residues. The Journal of Biological Chemistry 266(21): 13530-13536.

Von-Arx, J. 1981. The Genera of Fungi Sporulating in Pure Culture. 3 Ed. J. Crammer. Germany. 424 p.

Wang, Y., J. Li y J. Lin. 2008. Probiotics in aquaculture: challenges and outlook. Aquaculture 281(1): 1-4.

Ward-Rainey, N., F. Rainey y E. Stackebrandt. 1996. A study of the bacterial flora associated with Holothuria atra. Journal of Experimental Marine Biology and Ecology 203(1): 11-26.

Fecha de Recepción: 29/05/2014

Fecha de Aceptación: 22/10/2014

Para citar este artículo: Luna-Fontalvo, J., A, Rodriguez-Forero y J. Sarmiento-Rodriguez. 2014. Microbiota aislada del pepino de mar (Isostichopus badionotus) nativo de la bahía de Taganga, Caribe colombiano. Revista Intropica 9: 75 - 83 